\title{
Attribute-Sets: A Practically Motivated Enhancement to Attribute-Based Encryption
}

\author{
Rakesh Bobba, Himanshu Khurana and Manoj Prabhakaran \\ University of Illinois at Urbana-Champaign \\ \{rbobba,hkhurana,mmp\}@illinois.edu
}

July 27, 2009

\begin{abstract}
In distributed systems users need to share sensitive objects with others based on the recipients' ability to satisfy a policy. Attribute-Based Encryption (ABE) is a new paradigm where such policies are specified and cryptographically enforced in the encryption algorithm itself. Ciphertext-Policy ABE (CP-ABE) is a form of $\mathrm{ABE}$ where policies are associated with encrypted data and attributes are associated with keys. In this work we focus on improving the flexibility of representing user attributes in keys. Specifically, we propose Ciphertext Policy Attribute Set Based Encryption (CP-ASBE) - a new form of CP-ABE - which, unlike existing $\mathrm{CP}-\mathrm{ABE}$ schemes that represent user attributes as a monolithic set in keys, organizes user attributes into a recursive set based structure and allows users to impose dynamic constraints on how those attributes may be combined to satisfy a policy. We show that the proposed scheme is more versatile and supports many practical scenarios more naturally and efficiently. We provide a prototype implementation of our scheme and evaluate its performance overhead.
\end{abstract}

\section{Introduction}

In distributed systems users need to share sensitive objects with others based on the recipients' ability to satisfy a policy. Attribute-Based Encryption (ABE) ushers in a new paradigm where such policies are specified and cryptographically enforced in the encryption algorithm itself. Existing ABE schemes come in two complimentary forms, namely, Key-Policy ABE (KP-ABE) schemes and Ciphertext-Policy ABE (CP-ABE) schemes. In KP-ABE schemes [11, 12, 14, 16], as the name indicates, attribute policies are associated with keys and data is annotated with attributes. Only those keys associated with a policy that is satisfied by the attributes annotating the data are able to decrypt the data. In CP-ABE schemes [2, 6, 10, 13], on the other hand, attribute policies are associated with data and attributes are associated with keys. Only those keys whose associated attributes satisfy the policy associated with the data are able to decrypt it. 
$\mathrm{CP}-\mathrm{ABE}$ is more intuitive as it is similar to traditional access control model where data is protected with access policies and users with credentials satisfying the policy are allowed access to it. Among the various CP-ABE schemes proposed the one proposed by Bethencourt et al. [2], which we will hereafter refer to as BSW, is the most practical to date. It supports arbitrary strings as attributes, numerical attributes in keys and integer comparisons in policies and provides a means for periodic key refreshment. Furthermore, the authors have developed a software prototype with a friendly interface for integration in systems. However, BSW and other CP-ABE schemes are still far from being able to support the needs of modern enterprise environments, which require considerable flexibility in specifying policies and managing user attributes as well as increased efficiency. This is in part due to the fact that keys in current $\mathrm{CP}-\mathrm{ABE}$ schemes can only support user attributes that are organized logically as a single set; i.e., users can use all possible combinations of attributes issued in their keys to satisfy policies. This, we observe, imposes some undesirable restrictions which are outlined below.

First, this makes it both cumbersome and tedious to capture naturally occurring "compound attributes", i.e., attributes build intuitively from other (singleton) attributes, and specifying policies using those attributes. For example, attributes that combine a traditional organizational role with short-term responsibilities result in useful compound attributes; e.g., 'Faculty' in 'College of Engineering' serving as 'Committee Chair' of a 'University Tenure Committee' in 'Spring2009' are all valid attributes in their own right and are likely to be used to describe users. The only way to prevent users from combining such attributes in undesirable ways when using current $\mathrm{CP}-\mathrm{ABE}$ schemes is by appending the (singleton) attributes as strings; i.e., faculty_collegeOfEngineering_committeeChair_univTenureCommittee_Spring2009. But this approach has an undesirable consequence in that it makes it challenging to support policies that involve other combinations of singleton attributes used to build the compound attribute; e.g., policies targeting "all committee chairs in Spring2009" or "faculty serving on tenure committees". This is because the underlying crypto in CP-ABE schemes can only check for equality of strings and thus cannot extract the "faculty" or "committeeChair" attributes from a compound attribute such as the one described above.

Second, $\mathrm{CP}-\mathrm{ABE}$ schemes that support numerical attributes (i.e., allow numerical comparisons in policies) are limited to assigning only one value to any given numerical attribute within a key. But there are many real world systems where multiple numerical value assignments for a given attribute are common; $e . g$., students enrolled in multiple courses identified by numeric course numbers in a given semester, users with multiple accounts at a particular bank, disease codes for individual diseases and disease classes used widely in health care. Furthermore, the ability to compare across such multiple value assignments adds flexibility to policy specification. For example, consider a college student enrolled in two junior level courses, 357 and 373, and two senior level courses, 411 and 418 respectively. Without support for multiple numerical value assignments for a given attribute specifying policies to target students enrolled in senior level courses, such as "course number greater than or equal to 400 and less than 500" is tedious and cumbersome.

Our Contribution. In this work we propose Ciphertext-Policy Attribute-Set Based 
Encryption (CP-ASBE), a form of CP-ABE, that addresses the above limitations of $\mathrm{CP}-\mathrm{ABE}$ by introducing a recursive set based structure on attributes associated with user keys. Specifically CP-ASBE allows, 1) user attributes to be organized into a recursive family of sets and 2) policies that can selectively restrict decrypting users to use attributes from within a single set or allow them to combine attributes from multiple sets. Thus, by grouping user attributes into sets such that those belonging to a single set have no restrictions on how they can be combined, CP-ASBE can support compound attributes without sacrificing the flexibility to easily specify policies involving the underlying singleton attributes. Similarly, multiple numerical assignments for a given attribute can be supported by placing each assignment in a separate set.

While restricting users to use attributes from a single set during decryption can be thought of as a regular CP-ABE scheme, the challenge in constructing a CP-ASBE scheme is in selectively allowing users to combine attributes from multiple sets within a given key while still preventing collusion, i.e., preventing users from combining attributes from multiple keys. We provide a construction for a CP-ASBE scheme that builds on BSW and evaluate its performance through a prototype implementation. We show that our construction is secure against chosen-plaintext attacks in the generic group model. However, our construction can be efficiently extended to be secure against chosen-ciphertext attacks using a transformation like Fujisaki-Okamoto $[8,20]$ or the techniques of Canetti, Halevi and Katz [?] just like the BSW scheme [2].

The rest of this paper is organized as follows. Section 2 further motivates CPASBE. Section 3 discusses related work. In Section 4 we give some preliminaries. We present our construction and discuss its security in Section 5. In Section 6 we discuss efficiency of the scheme, give details of our prototype implementation and discuss performance. Section 7 concludes the paper and discusses future directions.

\section{Motivation}

The ability to group attributes into sets and to frame policies that can selectively restrict the decrypting key to use attributes belonging to the same set is a powerful feature more than one might realize initially. In this section we illustrate its versatility by solving various problems in different contexts which did not have any reasonably efficient solutions prior to this.

\subsection{Supporting Compound Attributes Efficiently}

While existing CP-ABE schemes offer unprecedented expressive power for addressing users, for several natural scenarios they are inadequate. We illustrate this with the following natural example and show how CP-ASBE provides a simple solution.

Consider attributes for students derived from courses they have taken. Each student has a set of attributes (Course, Year, Grade) for each course she has taken. In the following, consider a simple policy "Students who took a $300 \leq$ Course $<400$ in Year $\geq 2007$ and got Grade $>2$." Using a CP-ABE scheme for this is challenging because, for instance, a student can take multiple courses and obtain different grades in them. The policy circuit will have to ensure that she cannot mix together attributes from 
different sets to circumvent the policy. We point out a few possible options of using $\mathrm{CP}-\mathrm{ABE}$, but all unrealistic or unsatisfactory. The efficiency parameters considered are the number of designed attributes given to each student, and the size of the designed policy (a circuit, with designed attributes as inputs, for enforcing the policy).

- For each course that the student has taken, let there be a single designed (boolean) attribute that she gets (e.g. cyg:373_2008_4). But the designed policy will have to (unrealistically) anticipate all such attributes that will satisfy the policy (e.g., cyg:300_2007_3 or cyg:301_2007_3 or . . or cyg:399_2010_4).

- Anticipate (again, unrealistically) all possible policies that may occur which the student's attributes will satisfy, and give her compound boolean attributes corresponding to each of these policies (e.g., cyg:373_2008_4, cyg:373_2008, cyg:( $\geq 300) \_2008$, cyg:( $\left.\geq 400\right) \_2007$-or-cyg:( $\left.\left.\geq 300\right) \_2008 \_(\geq 3), \ldots\right)$. In this case our designed policy is minimal, with just an input gate (labeled by the attribute cyg: $\left.(\geq 300,<400)_{-}(\geq 2007)_{-}(>2)\right)$ and an output gate.

- Fix an upper bound on the number of courses a student could ever take, say 50 , and give all attributes indexed by a counter (e.g. Course\#1, Year\#1, Grade\#1 etc.); then the policy will have to incorporate several cases (e.g., $(400<$ Course\#1 $\geq 300$ and Year\#1 $\geq 2007$ and Grade\# $1>2$ ) or $\ldots$ or $(400<$ Course\#50 $\geq 300$ and Year\#50 $\geq 2007$ and Grade\#50 $>2$ )). This increases the policy size by a factor of 50 .

If a policy can refer to more than one course, all these approaches will lead to even more inefficiency or restrictions. In particular, in the third (and the most efficient) approach, if a policy refers to just two courses, the blow up will be by a factor of 2500 instead of 50 .

We stress that these are not the only possibilities when using CP-ABE. In general, by giving more attribute keys, the circuit complexity of the policies can be reduced (the first two options above being close to the two extremes). One could achieve slightly smaller policies by adding judiciously chosen auxiliary attributes and adding some structure to values taken by these attributes (for instance, in the third option above, one can let the counter monotonically increase with the course number). However, the resulting schemes are still unrealistically inefficient in terms of policy size and/or number of keys, and further makes attribute revocation even less efficient.

A CP-ASBE scheme can be used to overcome these issues by assigning multiple values to the group of attributes but in different sets. In our example, for each course that a student has taken, she gets a separate set of values for the attributes (Course, Grade, Year). Thus the number of designed attributes she receives is comparable to the number of natural attributes she has; further, the designed policy is comparable in size to that of a policy that did not enforce the requirement that attributes from different courses should not be mixed together. In short, using CP-ASBE, we can obtain efficient ciphertext policy encryption schemes for several scenarios where existing CP-ABE scheme are insufficient.

Expressiveness in terms of Attribute-Databases Supported. Some of the flexibility illustrated above can be understood by viewing the association of attributes to a user as 
an entry in a database table. In such a table - which we will call the attribute table each row stands for a user and each column (other than user identity) for an attribute. ${ }^{1}$ The policy associated with a cipher-text could be considered a query into this table, to identify all users whose attributes satisfy a certain predicate.

The expressive power of a $\mathrm{CP}-\mathrm{ABE}$ scheme is given by the class of queries into this table that the scheme can support. For instance, BSW CP-ABE [2] supports a large class of such queries. One challenge to increase the expressive power would be to broaden this class. However, there is another important dimension in which the expressive power of CP-ABE scheme can be improved, by supporting a more general class of attribute tables. The above description of CP-ABE required that each user ID appears in only one row in the table. (In other words, the user ID must be a "superkey" in the attribute table.) Of course, a table can be forced to have this property, but leading to large blow ups in the number of designed attributes that a user receives or the size of the designed policy. On the other hand, a CP-ASBE scheme can directly support a table with multiple rows per user: attributes in each row is given as a separate set.

\subsection{Supporting Multiple Value Assignments}

A major motivation for CP-ASBE is to support multiple value assignments for a given attribute in a single key. ${ }^{2}$ To illustrate this, suppose score is a 6-bit integer representing the score a user receives in a game. (The user may possess several other attributes in the system.) The user can play the game several times and receive several values for score. This numerical attribute will be represented by 12 boolean attributes: score_bit0_0, score_bit0_1, ..., score_bit6_0 and score_bit6_1, corresponding to the values 0 and 1 for the six bits in the binary representation of the value. Now consider a user who has two values of score, 33 (binary 100001) and 30 (binary 011110). By obtaining attributes for the bit values of these two numbers, the user gets all 12 boolean attributes, effectively allowing him to pretend to have any score he wants.

CP-ASBE solves this problem elegantly: each value assignment of the numerical attribute is represented in a separate set with six boolean attributes each (one for each bit position). Note that attributes other than score need not be repeated.

Application: Efficient revocation. ABE schemes suffer from lack of an effective revocation mechanism for keys that have been issued (just like IBE). To address this in $\mathrm{CP}-\mathrm{ABE}$ in a limited manner, Bethencourt et al. [2] propose adding an expiration_time attribute to a user's key indicating the time (i.e., a numerical value) until which the key is considered to be valid. Then a policy can include a check on the expiration_time attribute as a numerical comparison. However, in practice the validity period of sensitive attributes has to kept small to reduce the window of vulnerability when a key is compromised, e.g. a day, a week or a month. At the end of this period the entire key will have to be re-generated and re-distributed with an updated expiration time imposing a heavy burden on the key server and key distribution process.

\footnotetext{
${ }^{1}$ In the case of a "large universe" of attributes, the number of columns could be very large - say all strings of 256 bits - and the resulting sparse table will never be stored directly as a table. Our examples shall mostly use the small universe scenarios, though they extend to the large universe setting as well.

${ }^{2}$ Note that multiple values for an attribute is relevant only when the attribute in question is not a boolean attribute (in a monotonic policy).
} 
CP-ASBE solves this problem more efficiently. First, we observe that while key validity is limited because of the window of vulnerability, the actual attribute assignments change far less frequently. Second, we observe that it is possible to add attributes retroactively to a user key, both in BSW CP-ABE and CP-ASBE, if key server is able to maintain some state information about the user key. Then, by allowing multiple value assignments to the expiration_time attribute we can simply add a new expiration value to the existing key. Thus, while we require the key server to maintain some state we avoid the need to generate and distribute new keys on a frequent basis. This reduces the burden on the key server by a factor proportional to the average number of attributes in user keys.

\section{Related Work}

While the concepts and ideas related to Attribute-Based Encryption have been alluded to in literature as far back as $[4,7]$ Sahai and Waters [16] proposed what is considered the first ABE scheme. Their scheme supported policies with a single threshold gate. Furthermore, the threshold value $k$, and size of the gate $n$ used in a policy, are fixed during setup in their Large Universe construction. Pirretti et al., [15] showed how to overcome this limitation of fixed $k$ and $n$ and demonstrated the use of threshold access policies for two applications. Traynor et al., [19] further demonstrated its scalability by applying it to massive conditional access systems. Goyal et al., [11] first defined the two complimentary forms of ABE, namely, KP-ABE and CP-ABE, and provided a construction for a KP-ABE ${ }^{3}$ scheme. The proposed KP-ABE scheme supported all monotonic boolean encryption policies and was later extended by Ostrovsky et al., [14] to support non-monotonic boolean formulas.

Bethencourt et al., [2] gave the first construction for a CP-ABE scheme. Their construction supported all monotonic boolean encryption policies and the security of their scheme was argued in the generic group model. Cheung and Newport [6] gave the first standard model construction of CP-ABE scheme. While their scheme supported both positive and negative attributes it was limited to policies with single AND gates. Nishide et al., [13] extended the scheme in [6] to support policy secrecy. Goyal et al. gave the first standard model construction of CP-ABE scheme that could support flexible policies [10]. Their scheme can realize all non-monotonic boolean formulas. However, since it is constructed using a KP-ABE scheme of [11], it is inefficient and has bounded ciphertext, i.e., the size of supported policies is fixed at setup. Katz et al. proposed a KP-ABE scheme in [12] that can support flexible policies and achieve policy secrecy. This scheme can be used to realize CP-ABE schemes but such schemes have a bounded ciphertext. All the above ABE schemes are designed to work with one Attribute Authority (AA), a trusted entity that generates master parameters and distributes keys to users, and hence limited to a single domain. Chase extended [16] to multiple authorities in [5]. While most of the past work on CP-ABE schemes is focused on improving the expressibility of encryption policies and providing policy privacy ours is the first work to consider the flexibility of representing attributes in

\footnotetext{
${ }^{3}$ The scheme proposed in [16] can in retrospect be viewed as a KP-ABE scheme.
} 
keys. All CP-ABE schemes to date can only support a monolithic set of user attributes which makes them inflexible and inefficient to capture naturally occurring "compound attributes". Our CP-ASBE scheme is the first to organize user attributes in keys and allow users to impose dynamic constraints on how attributes can be combined to satisfy policies, allowing our scheme more flexibility and efficiency when supporting "compound attributes".

Support for numerical attributes was first discussed in [2]. While the technique may be applicable to other schemes none of the existing CP-ABE schemes can support multiple value assignments for a given numerical attribute within a single key. Our CP-ASBE scheme is the first scheme to do so allowing it to support applications where such attribute assignments are needed without sacrificing flexibility of range queries (i.e., numerical comparisons) in policies for those attributes.

\section{Preliminaries}

Bilinear Maps. Let $\mathbb{G}_{1}, \mathbb{G}_{2}, \mathbb{G}_{T}$ be cyclic (multiplicative) groups of order $p$, where $p$ is a prime. Let $g_{1}$ be a generator of $\mathbb{G}_{1}$, and $g_{2}$ be a generator of $\mathbb{G}_{2}$. Then $e$ : $\mathbb{G}_{1} \times \mathbb{G}_{2} \rightarrow \mathbb{G}_{T}$ is a bilinear map if it has the following properties:

1. Bilinearity: for all $u \in \mathbb{G}_{1}, v \in \mathbb{G}_{2}$ and $a, b \in \mathbb{Z}_{p}$, we have $e\left(u^{a}, v^{b}\right)=$ $e(u, v)^{a b}$.

2. Non-degeneracy: $e(g, h) \neq 1$.

Usually, $\mathbb{G}_{1}=\mathbb{G}_{2}=\mathbb{G}$. $\mathbb{G}$ is called a bilinear group if the group operation and the bilinear map $e$ are both efficiently computable.

Key Structure. In CP-ABE schemes, an encryptor specifies an access structure for a ciphertext which is referred to as the ciphertext policy. Only users with secret keys whose associated attributes satisfy the access structure can decrypt the ciphertext. In CP-ABE schemes so far, a user's key can logically be thought of as a set of elements each of which corresponds to an associated attribute, such that only elements within a single set may be used to satisfy any given ciphertext policy (i.e. collusion resistance). In our scheme however, we use a recursive set based key structure where each element of the set is either a set itself (i.e. a key structure) or an element corresponding to an attribute. We define a notion of depth for this key structure, which is similar to the notion of depth for a tree, that limits this recursion. That is, for a key structure with depth 2 , members of the set at depth 1 can either be attribute elements or sets but members of a set at depth 2 may only be attribute elements. The following is an example of a key structure of depth 2 :

$$
\{\text { CS-Department, Grad-Student, }\{\text { Course101, TA }\},\{\text { Course525, Grad-Student }\}\}
$$

The depth of key structures that can be supported by our scheme is a system parameter that should be decided at the time of setup. That is, if the system is setup with a depth parameter of 5 , keys of depth 5 or less can be supported. For ease of exposition, we will

describe our scheme for key structures of depth 2 . But as we show in the full version 
[?], our construction is easily generalized to support keys of any depth $d$ where $d$ is fixed at setup.

The key structure defines unique labels for sets in the key structure. For key structures of depth 2, just an index (arbitrarily assigned) of the set among sets at depth 2 is sufficient to uniquely identify the sets. Thus if there are $m$ sets at depth 2 then an unique index $i$ where $1 \leq i \leq m$ is (arbitrarily) assigned to each set. The set at depth 1 is referred to as set 0 or simply the outer set. If $\psi$ represents a key structure then let $\psi_{i}$ represent the $i$ th set in $\psi$. Individual attributes inherit the label of the set they are contained in and are uniquely defined by the combination of their name and their inherited label. That is, while a given attribute might appear in multiple sets it can appear only once in any set. In the above example, the outer set and \{Course525, Grad-Student $\}$ are assigned labels 0 and 2 respectively, and the two instances of the attribute GradStudent are distinguished by the unique combination of their inherited set label and attribute name, (0, Grad-Student) and (2, Grad-Student), respectively. By default, a user may only use attribute elements within a set to satisfy a given ciphertext policy. That is, a user with the key structure from the above example may combine individual attributes either from the outer set (i.e., $\{$ CS-Department, Grad-Student $\}$ ) or from the set $\{$ Course101, TA $\}$ or from the set $\{$ Course 525, Grad-Student $\}$ to satisfy the policy associated with a given ciphertext but may not combine attributes across the sets. However, an encryptor may choose to allow combining attributes from multiple sets to satisfy the access structure by designating translating nodes in the access structure as explained below.

Access Structure. We build on the access structure used in [2] which is a tree whose non-leaf nodes are threshold gates. Each non-leaf node of the tree is defined by its children and a threshold value. Let $n c_{x}$ denote the number of children and $k_{x}$ the threshold value of node $x$, then $0<k_{x} \leq n c_{x}$. When $k_{x}=1$, the threshold gate is an OR gate and when $k_{x}=n c_{x}$ it is an AND gate. The access tree also defines an ordering on the children of a node, i.e., they are numbered from 1 to $n c_{x}$. For node $x$ such a number is denoted by index $(x)$. Each leaf node $y$ of the tree is associated with an attribute which is denoted by $\operatorname{att}(y)$. Furthermore, the encrypting user may designate some nodes in an access tree as translating nodes. Their function will become clear as we discuss below the conditions under which a key structure is said to satisfy an access tree.

Let $\mathcal{T}$ be an access tree whose root node is $r$. Let $\mathcal{T}_{x}$ denote a subtree of $\mathcal{T}$ rooted at node $x$. Thus $\mathcal{T}_{r}$ is the same as $\mathcal{T}$. Now we will define the conditions under which a key structure $\psi$ is said to satisfy a given access tree $\mathcal{T}$ assuming there are no designated translating nodes in the access tree. We will then extend the definition to consider the presence of translating nodes. A key structure $\psi$ is said to satisfy the access tree $\mathcal{T}$ if and only if $\mathcal{T}(\psi)$ returns a non-empty set $S$ of labels. We evaluate $\mathcal{T}_{x}(\psi)$ recursively as follows. If $x$ is a non-leaf node we evaluate $\mathcal{T}_{x^{\prime}}(\psi)$ for all children $x^{\prime}$ of $x$. $\mathcal{T}_{x}(\psi)$ returns a set $S_{x}$ containing unique labels such that for every label $l b l \in S_{x}$ there exists at least one set of $k \geq k_{x}$ children such that for each child $x^{\prime}$ of these $k$ children $S_{x^{\prime}}$ contains the label $l b l$. If $x$ is a leaf node then the set $S_{x}$ returned by $\mathcal{T}_{x}(\psi)$ contains a label $l b l$ if and only if $a t t(x) \in \psi_{l b l}$. Thus a key structure is is said to satisfy an access tree if it contains at least one set that has all the attributes needed to satisfy the 
access tree. Note that attributes belonging to multiple sets in the key structure cannot be combined to satisfy the access tree.

However, if there are designated translating nodes in the access tree, the algorithm $\mathcal{T}(\psi)$ is modified as follows. The algorithm $\mathcal{T}_{x}(\psi)$ is the same as above when $x$ is a leaf node. When $x$ is a non-leaf node we evaluate $\mathcal{T}_{x^{\prime}}(\psi)$ for all children $x^{\prime}$ of $x$. $\mathcal{T}_{x}(\psi)$ returns a set $S_{x}$ containing unique labels such that for every label $l b l \in S_{x}$ there exists at least one set of $k \geq k_{x}$ children such that for each child $x^{\prime}$ of these $k$ children $S_{x^{\prime}}$ either contains the label $l b l$ or $x^{\prime}$ is a translation node and $S_{x^{\prime}} \neq \emptyset$. Thus, if node $x$ is a designated translating node then, even if the attribute elements used to satisfy the predicate represented by the subtree rooted at $x$ belong to a different set in the key structure than those used to satisfy the predicates represented by the siblings of $x$ the decrypting user is able to combine them to satisfy the predicate represented by the parent node of $x$.

Syntax of CP-ASBE Scheme. A CP-ASBE scheme consists of four algorithms, Setup, KeyGen, Encrypt and Decrypt. The algorithm Setup produces a master key and a public key for the scheme. KeyGen takes as input the master-key, a user's identity and an attribute set; it produces a secret key for the user. Encrypt takes as input the public key of the scheme, a message and an access tree, and outputs a ciphertext. Finally, Decrypt takes a ciphertext and a secret-key (produced by KeyGen), and if the access-tree used to construct the ciphertext is satisfied by the attribute set for which the secret-key was generated, then it recovers the message from the ciphertext.

Security of CP-ASBE Scheme. Our notion of message indistinguishability for CPASBE scheme against chosen-plaintext attacks is similar to that for CP-ABE schemes [2].

Setup. The challenger runs the Setup algorithm and gives public parameters, PK, to the adversary.

Phase 1. The adversary makes repeated queries for private keys corresponding to attribute sets $\mathbb{A}^{1}, \ldots, \mathbb{A}^{q_{1}}$.

Challenge. The adversary submits two equal length messages $M_{0}$ and $M_{1}$, and a challenge access structure $\mathcal{T}^{*}$ such that none of the private keys obtained in Phase 1 corresponding to attribute sets $\mathbb{A}^{1}, \ldots, \mathbb{A}^{q_{1}}$ satisfy the access structure. The challenger flips a random coin $b$, and encrypts $M_{b}$ under $\mathcal{T}^{*}$. The resulting ciphertext CT is given to the adversary.

Phase 2. Phase 1 is repeated with the restriction that none of the attribute sets $\mathbb{A}^{q_{1}+1}, \ldots, \mathbb{A}^{q}$ satisfy the access structure corresponding to the challenge.

Guess. The adversary outputs a guess $b^{\prime}$ of $b$.

The advantage of an adversary $\mathcal{A}$ in this game is defined as $\operatorname{Pr}\left[b^{\prime}=b\right]-\frac{1}{2}$. This game could easily be extended to include chosen-ciphertext attacks by allowing for decryption queries in Phase 1 and Phase 2.

Definition 1. A CP-ASBE scheme is secure against chosen-plaintext attacks if all probabilistic polynomial time adversaries have at most a negligible advantage in the game above. 


\section{Our CP-ASBE Construction}

A key challenge in designing $\mathrm{CP}-\mathrm{ABE}$ schemes is preventing users from pooling together their attributes. BSW CP-ABE achieves this by binding together all the attribute key components for each user with a random number unique to the user. Since in a CP-ASBE scheme one must prevent arbitrary combination of attributes belonging to different sets (even if they belong to the same user), a natural idea would be to similarly use a unique random number for binding together attribute key components for each set, in addition to using a random number for each user. However, a CP-ASBE scheme must also support specific combinations of attributes from different sets, as specified in an access-tree. The key idea in our construction is to include judiciously chosen additional values in the ciphertext (and in the key) that will allow a user to combine attributes from multiple sets all belonging to the same user. As it turns out, such a modification could introduce new subtle ways for multiple users to combine their attributes. Our construction shows how to thwart such attacks, using appropriate levels of randomization among different users' keys.

Let $\mathbb{G}_{0}$ be a bilinear group of prime order $p$ and let $g$ be a generator of $\mathbb{G}_{0}$. Let $e: \mathbb{G}_{0} \times \mathbb{G}_{0} \rightarrow \mathbb{G}_{1}$ denote a bilinear map. Let $H:\{0,1\}^{*} \rightarrow \mathbb{G}_{0}$ be a hash function that maps any arbitrary string to a random group element. We will use this function to map attributes described as arbitrary strings to group elements.

$\operatorname{Setup}(d=2)$. The setup algorithm chooses random exponents $\alpha, \beta_{i} \in \mathbb{Z}_{p} \forall i \in\{1,2\}$. The algorithm sets the public key and master key as:

$$
\begin{aligned}
\mathrm{PK} & =\left(\mathbb{G}, g, h_{1}=g^{\beta_{1}}, f_{1}=g^{\frac{1}{\beta_{1}}}, h_{2}=g^{\beta_{2}}, f_{2}=g^{\frac{1}{\beta_{2}}}, e(g, g)^{\alpha}\right) \\
\mathrm{MK} & =\left(\beta_{1}, \beta_{2}, g^{\alpha}\right)
\end{aligned}
$$

Note that to support key structures of depth $d, i$ will range from 1 to $d$.

$\operatorname{KeyGen}(\mathrm{MK}, \mathbb{A}, u)$. Here $u$ is the identity of a user and $\mathbb{A}=\left\{A_{0}, A_{1}, \ldots, A_{m}\right\}$ is a key structure. $A_{0}$ is the set of individual attributes in the outer set (i.e. set 0 ) and $A_{1}$ to $A_{m}$ are sets of attributes at depth 2 that the user has. Let $A_{i}=\left\{a_{i, 1}, \ldots, a_{i, n_{i}}\right\}$. That is, $a_{i, j}$ denotes the $j$-th attribute appearing in set $A_{i}$, and $n_{i}$ denotes the number of attributes in the set $A_{i}$. (Note that for different values of $(i, j), a_{i, j}$ can be the same attribute.) The key generation algorithm chooses a unique random number, $r^{\{u\}} \in \mathbb{Z}_{p}$, for user $u$. It then chooses a set of $m$ unique random numbers, $r_{i}^{\{u\}} \in \mathbb{Z}_{p}$, one for each set $A_{i} \in \mathbb{A}, 1 \leq i \leq m$. For set $A_{0}, r_{0}^{\{u\}}$ is set to be the same as $r^{\{u\}}$. It also chooses a set of unique random numbers, $r_{i, j}^{\{u\}} \in \mathbb{Z}_{p}$, one for each $(i, j), 0 \leq i \leq m, 1 \leq j \leq n_{i}$. The issued key is:

$$
\begin{aligned}
\mathrm{SK}_{u}=(\mathbb{A}, D & =g^{\frac{\left(\alpha+r^{\{u\}}\right)}{\beta_{1}}}, \\
D_{i, j} & =g^{r_{i}^{\{u\}}} \cdot H\left(a_{i, j}\right)^{r_{i, j}^{\{u\}}}, D_{i, j}^{\prime}=g^{r_{i, j}^{\{u\}}} \text { for } 0 \leq i \leq m, 1 \leq j \leq n_{i}, \\
E_{i} & \left.=g^{\frac{\left(r^{\{u\}}+r_{i}^{\{u\}}\right)}{\beta_{2}}} \text { for } 1 \leq i \leq m\right)
\end{aligned}
$$


Note that the operations on the exponents in the above equations are modulo the order of the group, which is prime. Hence division in the exponent is well-defined. We omit the mod for convenience. Elements $E_{i}$ enable translation from $r_{i}^{\{u\}}$ (i.e., set $A_{i}$ at depth 2) to $r^{\{u\}}$ (i.e., the outer or parent set $A_{0}$ at depth 1) at the translating nodes. Elements $E_{i}$ and $E_{i^{\prime}}$ can be combined as $E_{i} / E_{i^{\prime}}$ to enable translation from $r_{i^{\prime}}^{\{u\}}\left(i . e\right.$., set $A_{i^{\prime}}$ ) to $r_{i}^{\{u\}}$ (i.e., the set $A_{i}$ ) at the translating nodes. Similarly, for a key structure of depth $d$, there will elements that enable translation from a set at depth $d$ to its parent set at depth $d-1$ and they will use $\beta_{d}$ and random numbers corresponding to the appropriate sets.

$\operatorname{Encrypt}(\mathrm{PK}, \mathrm{M}, \mathcal{T}) . M$ is the message, $\mathcal{T}$ is an access tree. The algorithm associates a polynomial $q_{\tau}$ with each node $\tau$ (including the leaves) in the tree $\mathcal{T}$. These polynomials are chosen in the following way in a top-down manner, starting from the root node $\mathrm{R}$. For each internal node $\tau$ in the tree, the degree $d_{\tau}$ of the polynomial $q_{\tau}$ is set to be one less than the threshold value $k_{\tau}$ of that node, that is, $d_{\tau}=k_{\tau}-1$. For leaf nodes the the degree is set to be 0 . For the root node $R$ the algorithm picks a random $s \in Z_{p}$ and sets $q_{R}(0)=s$. Then, it chooses $d_{R}$ other points randomly to define the polynomial $q_{R}$ completely. For any other node $\tau$, it sets $q_{\tau}(0)=q_{\text {parent }(\tau)}($ index $(\tau))$ and chooses $d_{\tau}$ other points randomly to completely define $q_{\tau}$. Here parent $(\tau)$ denotes the parent node of $\tau$. Let $\mathbb{Y}$ denote the set of leaf nodes in $\mathcal{T}$. Let $\mathbb{X}$ denote the set of translating nodes in the access tree $\mathcal{T}$. Then the ciphertext $\mathrm{CT}$ returned is as follows:

$$
\begin{aligned}
\mathrm{CT}= & \left(\mathcal{T}, \tilde{C}=M \cdot e(g, g)^{\alpha \cdot s}, C=h_{1}^{s}, \bar{C}=h_{2}^{s}, \forall y \in \mathbb{Y}: C_{y}=g^{q_{y}(0)},\right. \\
& \left.C_{y}^{\prime}=H(\operatorname{att}(y))^{q_{y}(0)}, \forall x \in \mathbb{X}: \hat{C}_{x}=h_{2}^{q_{x}(0)}\right)
\end{aligned}
$$

Translating values $\hat{C}_{x}^{\prime} s$ together with $E_{i}{ }^{\prime} s$ in user keys allow translation between sets at a translating node $x$ as will be described in the Decrypt function. Note that the element $\bar{C}$ is the same as $\hat{C}_{r}$ where $r$ denotes the root node. A variant of the scheme would be where $\bar{C}$ is not included in the ciphertext but is only released at the discretion of the encrypting user as $\hat{C}_{r}$. This would restrict decrypting users to only use individual attributes in the outer set except when explicitly allowed by the encrypting user by designating translating nodes.

Decrypt $\left(\mathrm{CT}, \mathrm{SK}_{u}\right)$. Here we describe the most straightforward decryption algorithm without regard to efficiency. The decryption algorithm is a recursive algorithm similar to the tree satisfaction algorithm described in Section 4. The decryption algorithm first runs the tree satisfaction algorithm on the access tree with the key structure i.e., $\mathcal{T}(\mathbb{A})$, and stores the results of each of the recursive calls in the access tree $\mathcal{T}$. That is, each node $t$ in the tree is associated with a set $S_{t}$ of labels that was returned by $\mathcal{T}_{t}(\mathbb{A})$. If $\mathbb{A}$ does not satisfy the tree $\mathcal{T}$ then the decryption algorithm returns $\perp$. Otherwise the decryption algorithm picks one of the labels, $i$, from the set returned by $\mathcal{T}(\mathbb{A})$ and calls a recursive function DecryptNode $\left(\mathrm{CT}, \mathrm{SK}_{u}, t, i\right)$ on the root node of the tree. Here CT is the ciphertext $\mathrm{CT}=\left(\mathcal{T}, \tilde{C}, C, \forall y \in Y: C_{y}, C_{y}^{\prime}, \forall x \in X: \hat{C}_{x}\right), \mathrm{SK}_{u}$ is a private key, which is associated with a key structure denoted by $\mathbb{A}, t$ is a node from $\mathcal{T}$, and $i$ is a label denoting a set of $\mathbb{A}$. Note that the ciphertext CT now contains tree information that is augmented by the results from $\mathcal{T}(\mathbb{A})$. DecryptNode $\left(\mathrm{CT}, \mathrm{SK}_{u}, t, i\right)$ is defined as follows. 
If $t \in \mathbb{Y}$, i.e., node $t$ is a leaf node, then $\operatorname{DecryptNode}\left(\mathrm{CT}, \mathrm{SK}_{u}, t, i\right)$ is defined as follows. If $\operatorname{att}(t) \notin A_{i}$ where $A_{i} \in \mathbb{A}$ then $\operatorname{DecryptNode}\left(\mathbf{C T}, \mathrm{SK}_{u}, t, i\right)=\perp$. If $\operatorname{att}(t)=a_{i, j} \in A_{i}$ where $A_{i} \in \mathbb{A}$ then:

$$
\begin{aligned}
\operatorname{DecryptNode}\left(\mathrm{CT}, \mathrm{SK}_{u}, t, i\right) & =\frac{e\left(D_{i, j}, C_{t}\right)}{e\left(D_{i, j}^{\prime}, C_{t}^{\prime}\right)} \\
& =\frac{e\left(g^{r_{i}^{\{u\}}} \cdot H\left(a_{i, j}\right)^{r_{i, j}^{\{u\}}}, g^{q_{t}(0)}\right)}{e\left(g^{r_{i, j}^{\{u\}}}, H\left(a_{i, j}\right)^{q_{t}(0)}\right)}=e(g, g)^{r_{i}^{\{u\}}} \cdot q_{t}(0)
\end{aligned}
$$

Note that set from which the satisfying attribute $a_{i, j}$ was picked is implicit in the result $e(g, g)^{r_{i}^{\{u\}} \cdot q_{t}(0)}$ (i.e., indicated by $\left.r_{i}^{\{u\}}\right)$. When $t \notin \mathbb{Y}$, i.e., node $t$ is a non-leaf node, then DecryptNode $\left(\mathrm{CT}, \mathrm{SK}_{u}, t, i\right)$ proceeds as follows:

1. Compute $\mathbb{B}_{t}$ which is an arbitrary $k_{t}$ sized set of child nodes $z$ such that $z \in B_{t}$ only if either (1) label $i \in S_{z}$ or (2) label $i^{\prime} \in S_{z}$ for some $i^{\prime} \neq i$ and $z$ is a translating node. If no such set exists then return $\perp$.

2. For each node $z \in B_{t}$ such that label $i \in S_{z}$ call DecryptNode $\left(\mathbf{C T}, \mathbf{S K}_{u}, t, i\right)$ and store output in $F_{z}$.

3. For each node $z \in B_{t}$ such that $i^{\prime} \in S_{z}$ and $i^{\prime} \neq i$ call DecryptNode $\left(\mathrm{CT}, \mathrm{SK}_{u}, t, i^{\prime}\right)$ store output in $F_{z}^{\prime}$. If $i \neq 0$ then translate $F_{z}^{\prime}$ to $F_{z}$ as follows:

$$
\begin{aligned}
F_{z} & =e\left(\hat{C}_{z}, E_{i} / E_{i^{\prime}}\right) \cdot F_{z}^{\prime} \\
& =e\left(g^{\beta_{2} \cdot q_{z}(0)}, g^{\frac{r_{i}^{\{u\}}-r_{i^{\prime}}^{\{u\}}}{\beta_{2}}}\right) \cdot e(g, g)^{r^{\{u\}} i^{\prime} \cdot q_{z}(0)}=e(g, g)^{r^{\{u\}_{i}} \cdot q_{z}(0)}
\end{aligned}
$$

Otherwise, translate $F_{z}^{\prime}$ to $F_{z}$ as follows:

$$
F_{z}=\frac{e\left(\hat{C}_{z}, E_{i^{\prime}}\right)}{F_{z}^{\prime}}=\frac{e\left(g^{\beta_{2} \cdot q_{z}(0)}, g^{\frac{r^{\{u\}}+r_{i^{\prime}}^{\{u\}}}{\beta_{2}}}\right)}{e(g, g)^{r_{i^{\prime}}^{\{u\}}} \cdot q_{z}(0)}=e(g, g)^{r^{\{u\}}} \cdot q_{z}(0)
$$

4. Compute $F_{t}$ using polynomial interpolation in the exponent as follows:

$$
\begin{aligned}
& F_{t}=\prod_{z \in B_{t}} F_{z}^{\Delta_{k, B_{z}^{\prime}}(0)}, \quad \text { where } k=\operatorname{index}(z), B_{z}^{\prime}=\left\{\operatorname{index}(z): z \in B_{t}\right\} \\
& \text { and Lagrange coefficient } \Delta_{i, S}(x)=\prod_{j \in S, j \neq i} \frac{x-j}{i-j} \\
&= \begin{cases}e(g, g)^{r_{i}^{\{u\}}} \cdot q_{t}(0) & \text { when } i \neq 0 \\
e(g, g)^{r(u\}} \cdot q_{t}(0) & \text { when } i=0\end{cases}
\end{aligned}
$$

The output of DecryptNode $\left(\mathrm{CT}, \mathrm{SK}_{u}, r, i\right)$ function on the root node $r$ is stored in $F_{r}$. If $i=0$ we have $F_{r}=e(g, g)^{r^{r u\}} \cdot q_{r}(0)}=e(g, g)^{r^{\{u\}} \cdot s}$ otherwise we have $F_{r}=e(g, g)^{r_{i}^{\{u\}}} \cdot s$. If $i \neq 0$ then we compute $F$ as follows: 


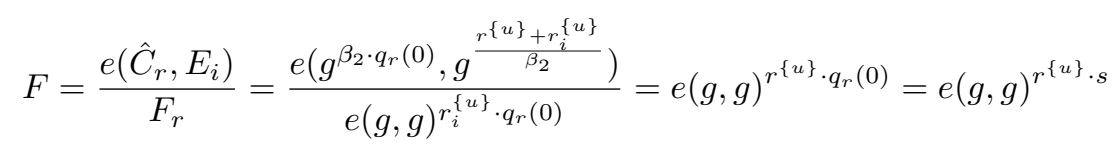

Otherwise $F=F_{r}$. The decryption algorithm then computes following:

$$
\frac{\tilde{C} \cdot F}{e(C, D)}=\frac{M \cdot e(g, g)^{\alpha \cdot s} \cdot e(g, g)^{r^{\{u\}} \cdot s}}{e\left(g^{s \cdot \beta_{1}}, g^{\left.\frac{(r\{u\}}{\beta_{1}}+\alpha\right)}\right)}=M
$$

Note how two elements $E_{i}$ and $E_{i^{\prime}}$ together with a translating value $\hat{C}_{t}$ at a node $t$ were used to translate between sets $i$ and $i^{\prime}$ at node $t$ in step 3. Similarly, note how a single element $E_{i}$ together with a translating value was used to translate between set $i$ and the outer set. We note that if $\beta_{1}=\beta_{2}$ then the scheme would become insecure as colluding users could transitively translate from inner set $i$ to outer set and then from one key to the other by using the $D$ elements from their keys. Thus we need a unique $\beta$ for every level that we need to support. When using key structures of depth $d$, translating values, $\hat{C}$ s, that help translate between sets at depth $d$ or between a set at depth $d$ and its parent at depth $d-1$ will use $\beta_{d}$. And to allow translations across multiple levels at a given node, multiple translating values using different $\beta \mathrm{s}$ will need to be released at that node.

Usage Example. We now demonstrate the usage of CP-ASBE with the example policy from Section 2.1. When using two level key structures, the policy can be written as follows using threshold gates:

$$
4 \text { OF } 4((\text { Course }>300),(\text { Course }<400),(\text { Grade }>2),(\text { Year }>2007))
$$

Here, predicates such as Course $>300$ will further be expanded and written using their constituent boolean attributes. Recall that numerical attributes in CP-ASBE are represented using a bag of bits representation, with a boolean attribute used to represent each bit of the numerical value, as described in Section 2.2. Users can be given keys with two levels. For example, for a user who has taken two courses the structure of the issued key is as follows:

$$
\{\{\text { Course }=304, \text { Grade }=2 \text {, Year }=2007\},\{\text { Course }=425, \text { Grade }=3 \text {, Year }=2008\}\}
$$

While the user's key will contain translation elements $E_{i}$ 's, as long as there is no designated translation node in the policy (i.e., ciphertext) the user will not be able to combine his Grade and Year attributes for Course 425 with that of Course 304 to satisfy the above policy. The form of the policy and keys for this example when using three level key structures is shown in the full version [?].

\subsection{Security}

The security proof for our scheme closely follows that of BSW CP-ABE [2] and uses generic group [3,18] and random oracle models [1]. Such a proof implies that the 
advantage of any adversary in the CP-ASBE security game is negligible, as long as it uses the underlying groups and hash functions in a generic manner, and makes only polynomially many accesses to them.

Generic Bilinear Group [3]. A generic group $\mathbb{G}_{0}$ with a bilinear map $e$ : $\mathbb{G}_{0} \times$ $\mathbb{G}_{0} \rightarrow \mathbb{G}_{1}$ can be modeled by an oracle which uses random strings as handles for the elements in the two groups $\mathbb{G}_{0}$ and $\mathbb{G}_{1} \cdot{ }^{4}$ More precisely, we consider an oracle $\mathcal{O}$, which picks two random encodings of the additive group $\mathbb{F}_{p}$ into sufficiently long strings, i.e., injective maps $\psi_{0}, \psi_{1}: \mathbb{F}_{p} \rightarrow\{0,1\}^{m}$, where $m>3 \log (p)$. We write $\mathbb{G}_{0}=\left\{\psi_{0}(x) \mid x \in \mathbb{F}_{p}\right\}$ and $\mathbb{G}_{1}=\left\{\psi_{1}(x) \mid x \in \mathbb{F}_{p}\right\}$. The oracle provides access to the group operations (which we shall refer to as multiplication) in either group: for example, queries of the form (multiply $\left.{ }_{0}, h, h^{\prime}\right)$ and (inverse $\left.{ }_{0}, h\right)$, will be answered respectively by $\psi_{0}\left(\psi_{0}^{-1}(h)+\psi_{0}^{-1}\left(h^{\prime}\right)\right), \psi_{0}\left(-\psi_{0}^{-1}(h)\right)$. If $h$ or $h^{\prime}$ is not in the range of $\psi_{0}$, then the oracle returns $\perp$. The oracle also provides access to the identity elements $\left(\psi_{0}(0), \psi_{1}(0)\right)$, and canonical generators $\left(\psi_{0}(1), \psi_{1}(1)\right)$ in the two groups, as well as the ability to sample random elements in the groups. In addition, given a query (pair, $h, h^{\prime}$ ), where $h=\psi_{0}(\alpha)$ and $h^{\prime}=\psi_{0}(\beta), \mathcal{O}$ returns $h^{\prime \prime}=\psi_{1}(\alpha \beta)$. To relate to the notation of bilinear groups used in our construction, we will denote $\psi_{0}(1)$ by $g$ and $\psi_{0}(x)$ by $g^{x}$. Similarly we will let $e(g, g)^{y}$ denote $\psi_{1}(y)$. Then the above pairing query to the oracle will be written as $e\left(g^{\alpha}, g^{\beta}\right)$ and the response as $e(g, g)^{\alpha \beta}$.

Finally, the oracle $\mathcal{O}$ also includes a random function $H:\{0,1\}^{*} \rightarrow \mathbb{G}_{0}$. It takes queries of the form (hash, $a$ ) for arbitrarily long strings $a$ and returns $H(a)$.

Theorem 2. Let $\mathcal{O}, \mathbb{G}_{0}, \mathbb{G}_{1}$, and $H$ be as defined above. For any adversary $\mathcal{A}$ with access to $\mathcal{O}$ in the security game for the CP-ASBE scheme in Section 5 (using $\mathbb{G}_{0}$, $\mathbb{G}_{1}$, and $\left.H\right)$, suppose $q$ is an upper-bound on the total number of group elements it receives from queries to $\mathcal{O}$ and interaction with the $C P-A S B E$ security game. Then the advantage of $\mathcal{A}$ in the $C P$-ASBE security game is $O\left(q^{2} / p\right)$.

Proof Intuition. Let us say that $s$ is the random secret split according to the access structure $\mathcal{T}$ as described in the Encrypt function of Section 5. Let $\mathcal{T}^{\prime}$ be an access structure derived from $\mathcal{T}$ by removing the sub-trees under all translating nodes, i.e., translating nodes become leaf nodes. For simplicity, let us assume for now that all the leaves of $\mathcal{T}^{\prime}$ are translating nodes in the original access structure $\mathcal{T}$. Let $q_{t}(0)$ represent the secret share associated with a translating node $t$. A user has to obtain $e(g, g)^{\alpha s}$ to recover the message encrypted using the access structure $\mathcal{T}$. He could pair $C=g^{\beta_{1} s}$ given in the ciphertext with $D=g^{\left(\alpha+r^{\{u\}}\right) / \beta_{1}}$ in his key to obtain $e(g, g)^{\alpha s+r^{\{u\}}} s$, i.e., $e(g, g)^{\alpha s}$ blinded by $e(g, g)^{r^{\{u\}} s}$. A user can cancel out $e(g, g)^{r^{\{u\}} s}$ only if he satisfies the tree, i.e., by obtaining a set of $e(g, g)^{r^{\{u\}}} q_{t}(0)$ that can reconstruct $e(g, g)^{r^{\{u\}} s}$. One can think of the key components given for each set of attributes in the key structure as a unique key under the BSW scheme. That is, if $r^{\{u\}}$ is the unique random number used in our CP-ASBE key then the set of key components (including the translation element) corresponding to each set $A_{i}$ can be thought of as a BSW key issued using a

\footnotetext{
${ }^{4}$ We remark that it is not important to model the handles as random strings, but only as distinct handles that can be named by the adversary. But we stick to the convention from [3], that was used in [2], whose proof ours most closely resemble.
} 
master secret key $\left(\beta_{2}, g^{r^{\{u\}}}\right)$. Furthermore, each of the sub-trees rooted at a translating node can be thought of an access structure under the BSW scheme. Thus a given subtree can only be satisfied using attributes from a single set, i.e. a single BSW key, as $\mathrm{BSW}$ is collusion resistant. The proof below shows that the additional group elements that are available to an adversary in our scheme do not adversely affect this collusion resistance. Thus a user who has a key with a set that can satisfy the sub-tree under a translating node $t$ can obtain $e(g, g)^{r^{\{u\}}} q_{t}(0)$. And since $r^{\{u\}}$ is unique to a CP-ASBE key, only attributes from sets within a single CP-ASBE key can be used to satisfy $\mathcal{T}^{\prime}$ and thus the original access structure.

Proof. Recall that in the CP-ASBE security game the adversary has to distinguish between challenge ciphertexts $M_{0} \cdot e(g, g)^{\alpha \cdot s}$ and $M_{1} \cdot e(g, g)^{\alpha \cdot s}$. By a standard hybrid argument one can consider a modified security game in which the adversary has to distinguish between challenge ciphertexts $e(g, g)^{\alpha \cdot s}$ and $e(g, g)^{\theta}$, where $\theta$ is selected uniformly at random form $\mathbb{F}_{p}$. It is easy to show that any adversary that has advantage $\epsilon$ in the original CP-ASBE game can be transformed into an adversary that has an advantage of at least $\epsilon / 2$ in the modified game. We will now bound the adversary's advantage in the modified game to prove the theorem.

For this we describe a simulation of the modified game, such that the adversary's view in the simulation is distributed identical to that in the modified security game with challenge ciphertext $e(g, g)^{\theta}$. Further, conditioned on an event of probability $1-O\left(q^{2} / p\right)$ in the simulation, we will show that this view is identical to what it would have been in the modified security game, with challenge ciphertext $e(g, g)^{\alpha \cdot s}$. Thus we will conclude that the advantage of the adversary is at most $O\left(q^{2} / p\right)$.

At the setup time the simulation chooses $\alpha, \beta_{1}, \beta_{2}$ at random from $\mathbb{F}_{p}$. If $\beta_{1}=0$ or $\beta_{2}=0$ then setup is aborted just as it would be in the actual scheme. The public parameters $h_{1}=g^{\beta_{1}}, h_{2}=g^{\beta_{2}}, f_{1}=g^{\frac{1}{\beta_{1}}}, f_{2}=g^{\frac{1}{\beta_{2}}}$, and $e(g, g)^{\alpha}$ are sent to the adversary.

When the adversary or the simulation call for evaluation of $H$ on any new attribute string $a$, a new random value $t_{a}$ is chosen from $\mathbb{F}_{p}$, and the simulation provides $g^{t_{a}}$ as the response to $H(a)$ and stores it to respond to future queries on $a$. When the adversary makes its $k^{\prime} t h$ key generation query for attribute set $\mathbb{A}^{k}$, the simulation picks, (1) a new random value $r^{\{k\}}$ from $\mathbb{F}_{p}$, (2) a new random value $r_{i}^{\{k\}}$ for every subset $A_{i}^{k} \in \mathbb{A}^{k}$ and (3) a new random value $r_{i, j}^{\{k\}}$ for every $a_{i, j} \in A_{i}^{k}$, for every $A_{i}^{k} \in \mathbb{A}^{k}$. It then computes, (1) $D=g^{\left(\alpha+r^{\{k\}}\right) / \beta_{1}}$, (2) $D_{i, j}=g^{r_{i}^{\{k\}}+t_{j} \cdot r_{i, j}^{\{k\}}}$ and $D_{i, j}^{\prime}=g^{r_{i, j}^{\{k\}}}$ for each $j \in A_{i}^{k}$ and each $A_{i}^{k} \in \mathbb{A}^{k}$ and (3) $E_{i}=g^{\left(r^{\{k\}}+r_{i, j}^{\{k\}}\right) / \beta_{2}}$ for each $A_{i}^{k} \in \mathbb{A}^{k}$. These values are passed on to the adversary.

In the challenge phase the adversary outputs a challenge access structure $\mathcal{T}^{*}$ along with two equal length messages $M_{O}, M_{1} \in \mathbb{G}_{0}$. Let $\mathbb{X}^{*}$ denote the set of converging nodes in $\mathcal{T}^{*}$. Let $\mathbb{Y}^{*}$ denote the set of leaf nodes in the access tree $\mathcal{T}^{*}$. The simulator chooses a random $s \in \mathbb{F}_{p}$ and uses the linear secret sharing scheme associated with $\mathcal{T}^{*}$ as described in Encrypt function of Section 5 to construct shares $q_{y}(0)$ of of $s \forall y \in \mathbb{Y}^{*}$. Let $\delta_{x}$ represent $q_{x}(0)$ for all $x \in \mathbb{X}^{*}$, where $q_{x}$ is polynomial associated with node $x$ as described in Encrypt function of Section 5. 
Note that every leaf node is a descendent of a unique converging node. Let $\lambda_{x, j}$ represent $q_{y}(0)$ where $y \in \mathbb{Y}^{*}$ is a descendent of $x \in \mathbb{X}^{*}$ and represents attribute $j^{5}$. Furthermore, note that the choice of $\delta_{x}$ can be perfectly simulated by choosing $v$ random values $\mu_{1}, \ldots, \mu_{v}$ uniformly and independently from $\mathbb{F}_{p}$, for some value of $v$, and then letting $\delta_{x}$ be fixed public linear combination of $\mu_{v}{ }^{\prime} s$ and $s$. Similarly, we can think of $\lambda_{x, j}{ }^{\prime} s$ as a fixed linear combination of some constants $\mu_{1}^{\prime}, \ldots, \mu_{u}^{\prime}$ and $\delta_{x}$. We will think of the $\delta_{x}{ }^{\prime} s$ and $\lambda_{x, j}{ }^{\prime} s$ as such linear combinations later.

Finally the simulation chooses a random $\theta \in \mathbb{F}_{p}$, and constructs the encryption as follows: $\tilde{C}=e(g, g)^{\theta}, C=h_{1}^{s}, \forall y \in \mathbb{Y}^{*}, C_{y}=g^{\lambda_{x, j}}$ and $C_{y}^{\prime}=g^{t_{j} \cdot \lambda_{x, j}}$, and $\forall x \in \mathbb{X}^{*} \hat{C}_{x}=h_{2}^{\delta_{x}}$. These values are sent to the adversary.

When the adversary makes a query to the group oracles, if the adversary provides as input a handle $h$ that it did not receive from the oracle, then with probability $1-$ $O\left(1 / p^{2}\right)$ such a handle is not in the range of $\psi_{0}$ or $\psi_{1}$. So, by conditioning on an event of probability $1-O\left(q / p^{2}\right)$ ( $q$ being an upperbound on the number of oracle queries made during the entire simulation), we can assume that the oracle provides answers to only queries which use handles already given out by the oracle. As such we may keep track of the algebraic expressions being called for from the oracles as long as no "accidental collisions" occur. Specifically, we think of an oracle query being a rational function $\nu=\eta / \xi$ in the variables $\theta, \alpha, \beta_{1}, \beta_{2}, t_{j}{ }^{\prime} s, r^{k^{\prime}} s, r_{i}^{k^{\prime}} s, r_{i, j}^{k} s, s$, and $\mu_{v}{ }^{\prime} s$. An accidental collision is said to occur if two distinct formal queries $\eta / \xi \neq \eta^{\prime} / \xi^{\prime}$ have the same value due to random choices of the variables. We now condition that no such "accidental collisions" in either group $\mathbb{G}_{0}$ or $\mathbb{G}_{1}$. For any pair of distinct formal queries $\eta / \xi$ and $\eta^{\prime} / \xi^{\prime}$ within a group, an accidental collision occurs only if the non-zero polynomial $\eta \xi^{\prime}-\xi \eta^{\prime}$ evaluates to zero. The total degree of $\eta \xi^{\prime}-\xi \eta^{\prime}$ in our case is a constant (at most 5). Then by the Schwartz-Zippel lemma [17, 21] the probability of an accidental collision between any pair of formal queries is $O(1 / p)$. By a union bound, the probability that any such collision happens in our simulation is at most $O\left(q^{2} / p\right)$. Thus we can condition on no "accidental collisions" occurring while retaining $1-O\left(q^{2} / p\right)$ of the probability mass.

Now we show that, subject to the condition that no "accidental collisions" occur, the view of the adversary is identically distributed when we set $\theta=\alpha \cdot s$ in the simulation. Since we are in the generic group model where each group element is uniformly and independently chosen the only way that the adversary's view can differ in the case $\theta=\alpha \cdot s$ is if there are two queries $\nu$ and $\nu^{\prime}$ into $\mathbb{G}_{1}$ such that $\nu \neq \nu^{\prime}$ but $\left.\nu\right|_{\theta=\alpha \cdot s}=$ $\left.\nu^{\prime}\right|_{\theta=\alpha \cdot s}$. Since $\theta$ only occurs as $e(g, g)^{\theta}$ in $\mathbb{G}_{1}$, the only dependence $\nu$ or $\nu^{\prime}$ can have on $\theta$ is by having some additive terms of the form $\gamma \cdot \theta$ where $\gamma$ is a constant. Therefore we must have that $\nu-\nu^{\prime}=\gamma \cdot \alpha \cdot s-\gamma \cdot \theta$ for some constant $\gamma \neq 0$ for the adversary's view to be different in the two simulations. We can then artificially add the query $\nu-\nu^{\prime}+\gamma \cdot \theta=\gamma \cdot \alpha \cdot s$ to the adversary's queries. We will now do a case analysis based on the information given to the adversary by the simulation to show that an adversary can never construct a query for $e(g, g)^{\gamma \cdot \alpha \cdot s}$ which will establish the theorem.

\footnotetext{
${ }^{5}$ Note we are assuming here that a given attribute is not represented by multiple leaf nodes descending from the same converging node. We can accommodate such policies by adding one more variable subscript to $\lambda$ that identifies its position among descendents of a given converging node. We omit it here for clarity.
} 
Table 1: Possible adversary queries terms in $\mathbb{G}_{1}$

\begin{tabular}{|c|c|c|c|}
\hline$r_{i, j}^{\{k\}}$ & $t_{j}$ & $r_{i}^{\{k\}}+t_{j} r_{i, j}^{\{k k\}}$ & $t_{j} \lambda_{x, j}$ \\
\hline$r_{i, j}^{\{k\}} r_{i^{\prime}, j^{\prime}}^{\left\{k^{\prime}\right\}}$ & $t_{j} t_{j^{\prime}}$ & $\lambda_{x, j} \lambda_{x^{\prime}, j^{\prime}}$ & $t_{j} t_{j^{\prime}} \lambda_{x, j} \lambda_{x^{\prime}, j^{\prime}}$ \\
\hline$r_{i, j}^{\{k\}} t_{j^{\prime}}$ & $t_{j} \lambda_{x, j^{\prime}}$ & $\lambda_{x, j} \lambda_{x^{\prime}, j^{\prime}} t_{j^{\prime}}$ & $\lambda_{x, j} t_{j} r_{i}^{\{k\}}+\lambda_{x, j} t_{j} t_{j^{\prime}} r_{i, j^{\prime}}^{\{k\}}$ \\
\hline$r_{i, j}^{\{k\}} \lambda_{x, j^{\prime}}$ & $t_{j} t_{j^{\prime}} \lambda_{x, j^{\prime}}$ & $r_{i}^{\{k\}} \lambda_{x, j^{\prime}}+t_{j} r_{i, j}^{\{k\}} \lambda_{x, j^{\prime}}$ & $r_{i, j}^{\{k\}} r_{i^{\prime}}^{\left\{k^{\prime}\right\}}+r_{i, j}^{\{k\}} t_{j^{\prime}} r_{i^{\prime}, j^{\prime}}^{\left\{k^{\prime}\right\}}$ \\
\hline$\alpha+r^{k}$ & $\delta_{x}$ & $r_{i}^{\{k\}} t_{j^{\prime}}+t_{j} t_{j^{\prime}} r_{i, j}^{\{k\}}$ & $r_{i, j}^{\{k\}} t_{j^{\prime}} \lambda_{x, j^{\prime}}$ \\
\hline$s$ & $r^{k}+r_{i}^{\{k\}}$ & $\alpha s+r^{k} s$ & $r^{k} \delta_{x}+r_{i}^{\{k\}} \delta_{x}$ \\
\hline$\lambda_{x, j}$ & \multicolumn{3}{c}{$\left(r_{i}^{\{k\}}+t_{j} r_{i, j}^{\{k\}}\right)\left(r_{i^{\prime}}^{\left\{k^{\prime}\right\}}+t_{j^{\prime}} r_{i^{\prime}, j^{\prime}}^{\left\{k^{\prime}\right\}}\right.$} \\
\hline
\end{tabular}

Table 1 enumerates all the possible queries into $\mathcal{G}_{1}$ by means of the bilinear map and group elements given to the adversary by the simulation except for those that involve $\beta_{1}$ or $\beta_{2}$ in every monomial as they will not be relevant for constructing a query involving $\alpha \cdot s$. Here the variables $j$ and $j^{\prime}$ are possible attribute strings, variables $k$ and $k^{\prime}$ are indices of secret key queries made by the adversary, and variables $i$ and $i^{\prime}$ are indices of attribute subsets in a given secret key. The queries are given in terms of $\lambda_{x, j}{ }^{\prime} s$ and $\delta_{x}{ }^{\prime} s$ and not in terms of $\mu_{v}{ }^{\prime} s$ and $\mu_{u}^{\prime}{ }^{\prime} s$. The adversary has access to 1 and $\alpha$ in group $\mathbb{G}_{1}$ in addition to the queries shown in Table 1 . The adversary can query for an arbitrary linear combination of these and we will show that no such linear combination can produce a polynomial of the form $\gamma \cdot \alpha \cdot s$ for some constant $\gamma \neq 0$.

From Table 1 we see that the only way for an adversary to create a term containing $\alpha s$ is by pairing $s \beta_{1}$ with $\left(\alpha+r^{\{k\}}\right) / \beta_{1}$ to get $\alpha s+r^{k} s$. The adversary could create a query polynomial of the form $\gamma \alpha s+\sum_{k \in T} \gamma_{k} r^{k} s$, for some set $T$ and constants $\gamma, \gamma_{k} \neq 0$. In order for the adversary to get a query polynomial of the form $\gamma \alpha s$ the adversary must add other terms in order to cancel the terms of $\sum_{k \in T} \gamma_{k} r^{k} s$. Observe that the only terms of the form $r^{k} s$ that the adversary has access to are obtained by pairing $\beta_{2} \delta_{x}$ terms with $\left(r^{k}+r_{i}^{\{k\}}\right) / \beta_{2}$ terms, since $\delta_{x}{ }^{\prime} s$ are linear combinations of $\mu_{v}{ }^{\prime} s$ and $s$. The adversary could create a query polynomial of the form for sets $T_{k}^{\prime}$ and constants $\gamma_{(i, k, x)} \neq 0$ :

$$
\gamma \alpha s+\sum_{k \in T}\left(\gamma_{k} r^{\{k\}} s+\sum_{(i, x) \in T_{k}^{\prime}} \gamma_{(i, k, x)}\left(r^{\{k\}} \delta_{x}+r_{i}^{\{k\}} \delta_{x}\right)\right)
$$

By design there exists at least one non empty set of $\delta_{x}{ }^{\prime} s$ that can reconstruct $s$. Without loss of generality we will assume that $\forall k \in T$ the adversary picks a set $T_{k}^{\prime}$ such that the set $\left\{\delta_{x} \mid(i, x) \in T_{k}^{\prime}\right\}$ can reconstruct $s$. (For otherwise the adversary's polynomial cannot be of the form $\gamma \alpha s$ thus proving the theorem.) The adversary still needs to add other terms to cancel terms of the form $\sum_{(i, x) \in T_{k}^{\prime}} \gamma_{(i, k, x)} r_{i}^{\{k\}} \delta_{x}, \forall k \in T$ in order to obtain a query polynomial of the form $\gamma \alpha \mathrm{s}$. Note that the only other terms of the form $r_{i}^{\{k\}} \delta_{x}$ that the adversary has access to are obtained by pairing $r_{i}^{\{k\}}+t_{j} r_{i, j}^{\{k\}}$ with some $\lambda_{x, j^{\prime}}$ as $\lambda_{x, j^{\prime}}$ is a linear combinations of $\mu_{u}{ }^{\prime} s$ and $\delta_{x}$. Thus the adversary 
could create a query polynomial of the following form:

$$
\begin{array}{r}
\gamma \alpha s+\sum_{k \in T}\left(\gamma_{k} r^{\{k\}} s+\sum_{(i, x) \in T_{k}^{\prime}} \gamma_{(k, i, x)}\left(r^{\{k\}} \delta_{x}\right)+\sum_{(i, x) \in T_{k}^{\prime}} \gamma_{(k, i, x)}\left(r_{i}^{\{k\}} \delta_{x}+\right.\right. \\
\left.\left.\sum_{\left(j, j^{\prime}\right) \in T_{k, i, x}^{\prime \prime}} \gamma_{\left(k, i, x, j, j^{\prime}\right)}\left(r_{i}^{\{k\}} \lambda_{x, j^{\prime}}+t_{j} r_{i, j}^{\{k\}} \lambda_{x, j^{\prime}}\right)\right)\right)
\end{array}
$$

The following case analysis concludes the proof:

Case 1: There exists some $k \in T: \exists(i, x) \in T_{k}^{\prime}$ such that the set of shares $L_{k, i, x}=$ $\left\{\lambda_{x, j^{\prime}}: \exists j:\left(j, j^{\prime}\right) \in T_{k, i, x}^{\prime \prime}\right\}$ do not allow for the reconstruction of $\delta_{x}$. In this case, the term $r_{i}^{\{k\}} \delta_{x}$ will not be canceled and the adversary's query polynomial cannot be of the form $\gamma \alpha s$.

Case 2: For all $k \in T$ and $\forall(i, x) \in T_{k}^{\prime}$ the set of shares $L_{k, i, x}=\left\{\lambda_{x, j^{\prime}}: \exists j\right.$ : $\left.\left(j, j^{\prime}\right) \in T_{k, i, x}^{\prime \prime}\right\}$ allow for the reconstruction of $\delta_{x}$. Then the only terms left in the adversary's query polynomial other than $\gamma \alpha s$ are of the form $t_{j} r_{i, j}^{\{k\}} \lambda_{x, j^{\prime}}$ and the adversary needs to add other terms to cancel them from the query. As seen in Table 1, the only term the adversary has access to that he can use to cancel terms of the form $t_{j} r_{i, j}^{\{k\}} \lambda_{x, j^{\prime}}$ is the term $r_{i, j}^{\{k\}} t_{j^{\prime}} \lambda_{x, j^{\prime}}$ but only when $j=j^{\prime}$. We will now show that there is at least one term of the form $t_{j} r_{i, j}^{\{k\}} \lambda_{x, j^{\prime}}$ in the adversary's query polynomial such that $j \neq j^{\prime}$ to complete the proof.

Fix any $k \in T$. Consider $\mathbb{A}^{k}$ the set of attributes corresponding to $k^{\prime} t h$ adversary key. By the assumption that no key should pass the challenge access structure, and the properties of the secret sharing scheme we know that there exists a $\delta_{x}$ : $\exists i:(i, x) \in T_{k}^{\prime}$ such that the set of shares $L_{k, i, x}^{\prime}=\left\{\lambda_{x, j}: j \in A_{i}^{k}: A_{i}^{k} \in \mathbb{A}^{k}\right\}$ cannot reconstruct $\delta_{x}$ for any $i: A_{i}^{k} \in \mathbb{A}^{k}$. Thus there must exist at least one $\lambda_{x, j^{\prime}} \in L_{k, i, x}$ that is linearly independent of $L_{k, i, x}^{\prime}$ when written in terms of $\delta_{x}$ and $\mu_{v}{ }^{\prime} s$. Thus for at least for one $\lambda_{x, j^{\prime}} \in L_{k, i, x}$ there will be a term of the form $t_{j} r_{i, j}^{\{k\}} \lambda_{x, j^{\prime}}: j \neq j^{\prime}$ left behind in the query for the adversary does not have access to a term that can cancel it as evident from Table 1. Therefore no adversary query polynomial can be of the form $\gamma \alpha s$.

\section{Evaluation}

In this section we discuss the efficiency of CP-ASBE scheme instantiated with twolevels, describe its implementation and evaluate its performance overhead relative to BSW CP-ABE.

Efficiency. It is straightforward to estimate the efficiency of our key generation and encryption algorithms. In terms of computation, our key generation algorithm requires 
two exponentiations for every attribute in the key issued to the user and two exponentiations for every set (including recursive sets for a scheme with levels $>2$ ) in the key. In terms of key size, the private key contains two group elements per attribute and one group element per attribute set. Compared to BSW the additional key generation cost is two exponentiations for every attribute set in terms of computation and one group element per attribute set in terms of size. Encryption involves two exponentiations per leaf node in the tree and one exponentiation per translating node in the tree. The ciphertext contains two group elements per leaf node and one group element per translating node. Compared to BSW the additional cost is one exponentiation per translating node in terms of computation and one group element per translating node in terms of size. The cost of decrypting a given ciphertext however varies depending on the key used for decryption. Even for a given key there might be multiple ways to satisfy the associated access tree. The decrypt algorithm needs, 1) two pairings for every leaf node used to satisfy the tree, 2) one pairing for every translating node on the path from the leaf node used to the root and 3) one exponentiation for every node on the path from the leaf node to the root. However, by employing the optimization technique of flattening the recursive calls to DecryptNode, as described in BSW [2] albeit modified to accommodate translating nodes, we can reduce the cost to 1) two pairings and one exponentiation per leaf node used and 2) one pairing and one exponentiation per translating node on the path from a used leaf node to the root. Compared to BSW the additional cost is one pairing and one exponentiation per translating node on the path from a used leaf node to the root. In a multi-level (level $>2$ ) instantiation the overhead will be per translation rather than per translating node as multiple translations may be needed at a given translating node for such instantiations.

Implementation. We have implemented a two-level CP-ASBE scheme as described in Section 5. The only difference is that the implemented decryption function is optimized to improve the efficiency and performance.

Our implementation leverages the cpabe toolkit (http: / / a csc.csl.sri.com/ cpabe/) developed for BSW which uses the Pairing-Based Cryptography library (http://crypto.stanford.edu/pbc/). The interface for the cpasbe toolkit is similar to that of cpabe toolkit and is as follows:

cpasbe-setup Generates a public key and a master key.

cpasbe-keygen Given a master key, generates a private key for a given set of attributes; compiles numerical attributes into 'bag of bits' representation and treats the resulting attributes as a 'set'.

cpasbe-enc Given a public key, encrypts a file under a given access policy; numerical comparisons in the policy are represented by access sub-trees comprising 'bag of bits' representation of the numerical attribute with the root node of the sub-tree treated as a translating node.

cpasbe-dec Decrypts a file, given a private key.

The cpasbe toolkit is similar to cpabe toolkit in that it supports numerical attributes and range queries (i.e., numerical comparisons) in access policies. However, unlike in 


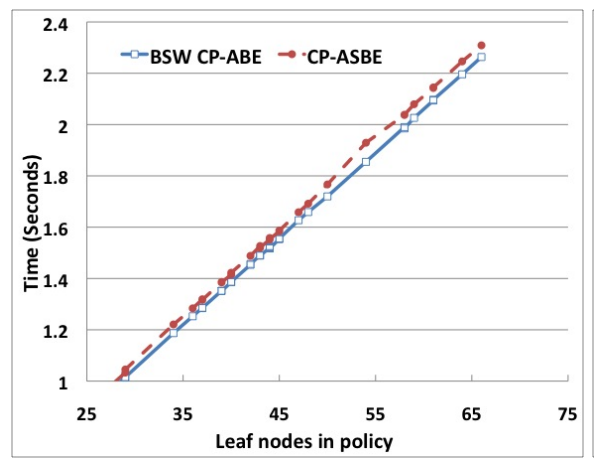

(a) Encryption Time

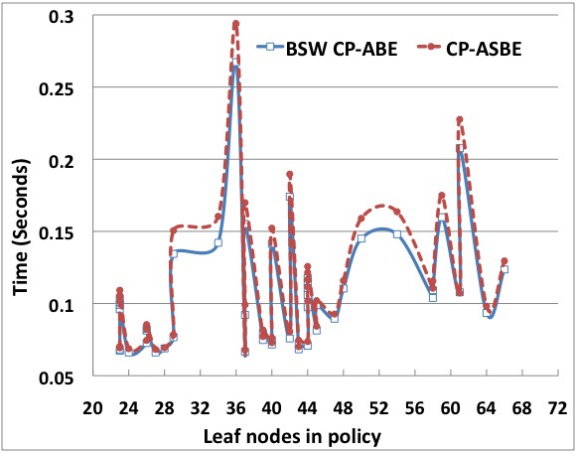

(b) Decryption Time

Figure 1: Encryption and Decryption Times

cpabe toolkit, numerical attributes in cpasbe are treated as sets and thus cpasbe toolkit supports multiple numerical value assignments to a given attribute in a single private key. Thus a user with a private key generated using the following command cannot claim any score other than 33 and 30.

$\$$ cpasbe-keygen -o tom-priv-key pub-key master-key 'score $=33$ ' 'score $=30$ ' tom

Performance Overhead. A two-level CP-ASBE scheme provides better functionality over $\mathrm{CP}-\mathrm{ABE}$ schemes in terms of, 1) better supporting compound attributes and 2) supporting multiple numerical value assignments for a given attribute in a single key. In order to gauge the cost of this additional functionality we compared the encryption, decryption and key generation times using randomly generated policies and associated keys with those of BSW CP-ABE scheme. The policies used to encrypt data were randomly generated formulae in the disjunctive normal form with the number of leaf nodes ranging from 23 to 66 . For each policy, a representative set of keys that satisfy the policy are generated and used for decryption. Specifically, 1) a key is generated for each conjunctive clause in the policy such that it satisfies the clause and 2) a key is generated for each combination of conjunctive clauses in the policy such that the key satisfies all the clauses in the combination. The generated keys had boolean attributes, ranging from 1 to 422 , i.e., including the "bag of bits" representation for numbers with 64 bits used to represent each integer. Decryption time for a policy is the average of decryption times with all the keys generated for that policy as described above. Experiments were run on a Linux box with quad core 3.0 Ghz Intel Xeon and 2GB of RAM. Both implementations used a 160-bit elliptic curve group constructed on the curve $y^{2}=x^{3}+x$ over a 512-bit field.

While key generation time are not shown due to space constraints, as expected, they were found to be linear in the number of attributes in the key, and CP-ASBE imposed very little overhead over BSW CP-ABE. On an average, CP-ASBE imposed $18 \mathrm{~ms}$ overhead per numerical attribute, i.e., per set, in the key and no overhead when there are no numerical attributes. To put this overhead in perspective, generating a key with 2 numerical attributes (and 145 boolean attributes in total) took $5 s$ seconds when using BSW CP-ABE scheme and 5.035s when using CP-ASBE scheme. Encryption and decryption times are shown in Figure 1. Encryption time is, as expected, linear in the number of leaves in the policy tree, and CP-ASBE imposed very little overhead when 
compared to BSW CP-ABE. On an average, CP-ASBE imposed $8.3 m s$ overhead per translating node in the policy. Since decryption time is dependent on both the structure of the policy tree and the key used for decryption, it varied significantly even for a given policy size. However, in this case too CP-ASBE scheme imposed very little to no overhead over BSW CP-ABE, $6.7 \mathrm{~ms}$ on average. Overhead results are consistent with our efficiency analysis and performance numbers in general are consistent with those reported in [2].

\section{Conclusion and Future Work}

In this work we proposed CP-ASBE a form of CP-ABE that organizes user attributes into a recursive family of sets and allows users to impose dynamic constraints on how attributes may be combined. We demonstrated how CP-ASBE can naturally support compound attributes, and numerical attributes with multiple value assignments. We showed that it achieves this versatility with very little overhead through efficiency analysis and performance evaluation of a prototype implementation. An interesting direction for future research is to study the potential of CP-ASBE schemes and ABE schemes in general in supporting constructs similar to "OR roles" [9] and constraints like "dynamic mutually exclusive roles" that are common in traditional mediated RBAC settings. Other directions for future work are the design of efficient CP-ASBE schemes that are secure in the standard model and extending CP-ASBE to a multi-authority setting.

\section{References}

[1] M. Bellare and P. Rogaway. Random oracles are practical: A paradigm for designing efficient protocols. In ACM Conference on Computer and Communications Security, pages 62-73, 1993.

[2] J. Bethencourt, A. Sahai, and B. Waters. Ciphertext-policy attribute-based encryption. In IEEE Symposium on Security and Privacy, 2007.

[3] D. Boneh, X. Boyen, and E.-J. Goh. Hierarchical identity based encryption with constant size ciphertext. In R. Cramer, editor, EUROCRYPT, volume 3494 of Lecture Notes in Computer Science, pages 440-456. Springer, 2005.

[4] D. Boneh and M. Franklin. Identity-Based Encryption from the Weil Pairing. Advances in Cryptology-Crypto 2001: 21st Annual International Cryptology Conference, Santa Barbara, California, USA, August 19-23, 2001, Proceedings, 2001.

[5] M. Chase. Multi-authority attribute based encryption. In S. P. Vadhan, editor, TCC, volume 4392 of Lecture Notes in Computer Science, pages 515-534. Springer, 2007. 
[6] L. Cheung and C. Newport. Provably secure ciphertext policy abe. In CCS '07: Proceedings of the 14th ACM conference on Computer and communications security, pages 456-465, New York, NY, USA, 2007. ACM.

[7] C. Cocks. An identity based encryption scheme based on quadratic residues. In B. Honary, editor, IMA Int. Conf., volume 2260 of Lecture Notes in Computer Science, pages 360-363. Springer, 2001.

[8] E. Fujisaki and T. Okamoto. Secure Integration of Asymmetric and Symmetric Encryption Schemes. In M. J. Wiener, editor, CRYPTO, volume 1666 of Lecture Notes in Computer Science, pages 537-554. Springer, 1999.

[9] L. Giuri. A New Model for Role-Based Access Control. In Annual Computer Security Application Conference, pages 249-255, December 1995.

[10] V. Goyal, A. Jain, O. Pandey, and A. Sahai. Bounded Ciphertext Policy Attribute Based Encryption. In L. Aceto, I. Damgård, L. A. Goldberg, M. M. Halldórsson, A. Ingólfsdóttir, and I. Walukiewicz, editors, ICALP (2), volume 5126 of Lecture Notes in Computer Science, pages 579-591. Springer, 2008.

[11] V. Goyal, O. Pandey, A. Sahai, and B. Waters. Attribute-based encryption for fine-grained access control of encrypted data. In ACM Conference on Computer and Communications Security, pages 89-98, 2006.

[12] J. Katz, A. Sahai, and B. Waters. Predicate Encryption Supporting Disjunctions, Polynomial Equations, and Inner Products. In N. P. Smart, editor, EUROCRYPT, volume 4965 of Lecture Notes in Computer Science, pages 146-162. Springer, 2008.

[13] T. Nishide, K. Yoneyama, and K. Ohta. Attribute-based encryption with partially hidden encryptor-specified access structures. In S. M. Bellovin, R. Gennaro, A. D. Keromytis, and M. Yung, editors, ACNS, volume 5037 of Lecture Notes in Computer Science, pages 111-129, 2008.

[14] R. Ostrovsky, A. Sahai, and B. Waters. Attribute-based encryption with nonmonotonic access structures. In ACM Conference on Computer and Communications Security, 2007.

[15] M. Pirretti, P. Traynor, P. McDaniel, and B. Waters. Secure attribute-based systems. In ACM Conference on Computer and Communications Security, pages 99-112, 2006.

[16] A. Sahai and B. Waters. Fuzzy identity-based encryption. In R. Cramer, editor, EUROCRYPT, volume 3494 of Lecture Notes in Computer Science, pages 457473. Springer, 2005.

[17] J. T. Schwartz. Fast probabilistic algorithms for verification of polynomial identities. J. ACM, 27(4):701-717, 1980. 
[18] V. Shoup. Lower bounds for discrete logarithms and related problems. In EUROCRYPT, Lecture Notes in Computer Science, pages 256-266, 1997.

[19] P. Traynor, K. Butler, W. Enck, and P. McDaniel. Realizing massive-scale conditional access systems through attribute-based cryptosystems. In Proceedings of The 15th Annual Network and Distributed System Security Symposium (NDSS), February 2008.

[20] P. Yang, T. Kitagawa, G. Hanaoka, R. Zhang, K. Matsuura, and H. Imai. Applying Fujisaki-Okamoto to Identity-Based Encryption. In M. P. C. Fossorier, H. Imai, S. Lin, and A. Poli, editors, AAECC, volume 3857 of Lecture Notes in Computer Science, pages 183-192. Springer, 2006.

[21] R. Zippel. Probabilistic algorithms for sparse polynomials. In E. W. Ng, editor, EUROSAM, volume 72 of Lecture Notes in Computer Science, pages 216-226. Springer, 1979. 